\section{Christian Bonnerot}

\section{Hervé Fridman}

\title{
Bases moléculaires de la diversité fonctionnelle des récepteurs des anticorps
}

Les récepteurs de faible affinité de la portion Fc des

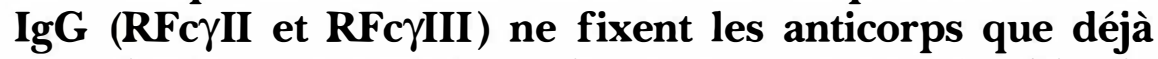
complexés à un antigène. Ils constituent une famille de glycoprotéines membranaires très homologues dans leurs régions extracellulaires mais complètement différentes dans leurs régions transmembranaire et intracytoplasmique. Ces récepteurs relaient les très diverses activités biologiques des anticorps à la surface des cellules du système immunitaire : à la surface des macrophages, des neutrophiles et des cellules NK, les RFc $\gamma$ déclenchent l'activation cellulaire, intervenant dans des phénomènes de cytotoxicité dépendante d'anticorps ; à la surface des mastocytes, les RFc $\gamma$ sont capables de déclencher la dégranulation de ces cellules, et donc aussi la libération de médiateurs de l'inflammation, pouvant intervenir dans les phénomènes d'allergie; à la surface des lymphocytes $\mathrm{B}$, le pontage des immunoglobulines membranaires avec les RFcyII bloque l'activation cellulaire et module ainsi la synthèse d'immunoglobulines. Dans les macrophages, l'interaction des récepteurs $\mathrm{RF} \gamma$ avec leurs ligands provoque l'internalisation du complexe; ces récepteurs participent donc à la clairance des complexes immuns et à l'internalisation de l'antigène qu'ils contiennent.

\section{ADRESSE}

C. Bonnerot : chargé de recherche à l'Inserm. $\mathrm{H}$. Fridman: directeur de recherche à l'Inserm. Inserm U.255, Institut Curie, 26, rue d’Ulm, 75005 Paris, France.

TIRÉS A PART

C. Bonnerot.

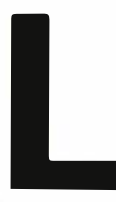

es anticorps sont le siège d'une double diversité dont l'une permet la reconnaissance d'une multitude d'antigènes et l'autre détermine leur spécificité fonctionnelle. La région variable des anticorps est codée par un ensemble d'une centaine de gènes dont les différentes possibilités de recombinaison sont à l'origine du répertoire des lymphocytes B. Les parties variables s'associent aux régions constantes, elles-mêmes codées par une dizaine de gènes, qui définissent les classes isotypiques d'immu- noglobulines (Ig) M, D, G, E et A, diversité moléculaire localisée principalement dans la portion Fc des anticorps. A partir de la description des nombreuses activités biologiques des anticorps a émergé la vieille notion d'anticorps cytophile, c'est-àdire d'anticorps ayant la capacité de se fixer sur les cellules. Beaucoup plus tard, la description à la surface de la plupart des cellules du système immunitaire de récepteurs de la portion Fc des anticorps a permis de mieux connaître les molécules qui assurent la connexion entre immunité cellulaire et immunité humo- 
rale. Des récepteurs de la portion Fc de chacune des classes et sous-classes d'immunoglobulines ont ainsi été décrits, mais les mieux connus restent les récepteurs des IgE et des IgG dénommés respectivement RFcE et RFcy. L'affinité de ces récepteurs pour leurs ligands a abouti à la définition fonctionnelle de deux grands groupes de RFc. Les RFc de forte affinité (ou de type I) fixent les anticorps sous forme monomérique, puis la complexation par l'antigène déclenche les processus d'activation cellulaire. Les RFcEI ou les RFc $\gamma \mathrm{I}$ sont des exemples de ce type d'anticorps, le mieux connu étant le RFcEI des mastocytes qui est impliqué dans les phénomènes d'allergie, tandis que le RFc $\gamma \mathrm{I}$ des macrophages et des monocytes, appelé CD64 chez l'homme, n'a pas encore de fonction bien caractérisée. A l'opposé, les RFc de faible affinité ne fixent des anticorps que déjà préalablement complexés à un antigène, déclenchant directement les phénomènes d'activation cellulaire. Pour les IgE, le récepteur le mieux connu est la molécule CD23, tandis que pour les IgG, on distingue les récepteurs de type II et de type III appelés aussi CD32 et CD16.

\section{Structure des RFc $\gamma$ de faible affinité}

Les récepteurs de la portion $\mathrm{Fc}$ des IgG sont des glycoprotéines membranaires qui ont une organisation structurale commune. Ils appartiennent à la superfamille des immunoglobulines au même titre que les molécules $\mathrm{CD} 2, \mathrm{CD} 4, \mathrm{CD} 8$, les antigènes de classe I ou de classe II du complexe majeur d'histocompatibilité (CMH), le récepteur du PDGF, le récepteur des IgA polymériques, le récepteur de l'antigène des cellules $T$ (TcR). Ces molécules sont caractérisées par l'organisation de leur région extracellulaire en un ou plusieurs domaines homologues. Les RFc $\gamma$ de type II (RFc $\gamma I I)$ et les RFc $\gamma$ de type III (RFcyIII) sont très homologues; ils ont en effet la même spécificité isotypique et sont reconnus par le même anticorps monoclonal [1] chez la souris, tandis que, chez l'homme, un ensemble d'anticorps monoclonaux a permis de les différencier.

$m / s n^{\circ} 11$ vol. 9, novembre 93

\section{Les RFcyll}

Les RFcyII murins sont des molécules membranaires monocaténaires dont on connaît deux isoformes appelées RFc $\gamma$ IIbl et RFc $\gamma$ IIb2 qui ne diffèrent que par leurs régions intracytoplasmiques. Le RFc $\gamma$ IIb2 est constitué d'un domaine intracytoplasmique de 47 acides aminés alors que le RFcyIIbl a un domaine intracytoplasmique de 93 acides aminés par insertion de 46 acides aminés supplémentaires au niveau juxtamembranaire [2] (figure 1). Chez l'homme, on distingue trois récepteurs de type II dénommés RFc $\gamma$ IIA, RFc $\gamma$ IIB et RFc $\gamma$ IIC. Les RFc $\gamma$ IIB sont les plus proches des RFcyII murins. La conservation de séquence est de l'ordre de $80 \%$ et deux isoformes, B1 et B2, sont engendrées par épissage alternatif du premier exon codant pour le domaine intracytoplasmique comme cela a été décrit chez la souris [3] (figure 1). Les RFc $\gamma$ IIA et IIC sont presque identiques puisqu'ils ne diffèrent que par la substitution de quelques acides aminés dans le premier domaine extracellulaire et par la séquence de leurs peptides signals [3]. Leur homologie avec les RFc $\gamma I I B$ est importante dans les régions transmembranaire et extracellulaire mais ils comportent, en revanche, une région intracytoplasmique complètement différente (figure 1). Les mêmes 83 acides aminés constituent le domaine intracytoplasmique des RFc $\gamma$ IIA et IIC.

\section{Les RFCyIII}

Chez la souris, les récepteurs de type III ont été définis comme des complexes multimoléculaires où sont associés deux ou trois polypeptides différents en fonction du type cellulaire dans lequel ils s'expriment (figure 1). Ces chaînes ont été nommées $\alpha, \beta, \gamma$. La chaîne $\alpha$ est un élément constitutif spécifique du $\mathrm{RF} c \gamma \mathrm{III}$ responsable de la liaison des IgG [2]. Elle est composée de deux domaines extracellulaires, un segment transmembranaire et une courte portion intracytoplasmique. Les domaines extracellulaires de la chaîne $\alpha$ et des RFcyII sont homologues à $95 \%$; en revanche, les autres régions des deux récepteurs sont complètement différentes [2]. Les chaînes $\beta$ et $\gamma$ rentrent aussi dans la constitution d'autres complexes membranaires comme le RFcEI [4], pour les chaînes $\beta$ et $\gamma$, et le TcR [5], pour la chaîne $\gamma$. La chaîne $\beta$ a été initialement identifiée comme une des chaînes constituant le RFceI. Son expression étant restreinte au seul mastocyte, elle ne rentre dans la constitution des RFcyIII que pour ce type cellulaire [6].

Identifiés dans la nomenclature internationale comme CD16, on distingue chez l'homme deux RFc $\gamma$ de type III qui diffèrent par leur structure et leur mode d'ancrage membranaire ainsi que par leur distribution tissulaire. Ils ont été dénommés RFc $\gamma$ IIIA et RFc $\gamma$ IIIB. Le RFc $\gamma$ IIIA est un complexe transmembranaire qui a la même structure et la même distribution tissulaire que le récepteur de type III murin [7] (figure 1). Mais contrairement à ce dernier, il est le siège d'une diversité supplémentaire puisqu'il peut se composer soit d'un homodimère de chaînes $\gamma$ ou de chaînes $\zeta$ du TcR, soit d'un hétérodimère $\gamma \zeta$ [8]. Par ailleurs, la chaîne $\beta$ du RFcEI peut aussi s'associer au RFc $\gamma$ IIIA engendrant ainsi un autre niveau de diversité [6] (figure 1). Le RFcyIIIB n'est présent que chez l'homme et est ancré à la membrane des neutrophiles par un phosphatidylinositol après clivage des domaines transmembranaire et intracytoplasmique [9]. Les chaînes $\alpha$ des RFc $\gamma$ IIIB et IIIA sont homologues à $98 \%$ : elles ne comportent que quelques différences ponctuelles [7]. Pour un RFc $\gamma$ III $\alpha, \gamma 2$ chez la souris, il existe donc quatre formes de récepteur possibles chez l'homme (RFc $\gamma$ IIIA $\alpha, \gamma 2$, RFc $\gamma$ IIIA $\alpha, \zeta 2$, RFc $\gamma$ IIIA $\alpha, \gamma-\zeta$, et RFc $\gamma I I I B)$ auxquelles viennent s'ajouter les possibilités d'association à la chaîne $\beta$ à la surface des mastocytes.

\section{Rôles biologiques des RFc $\gamma$ II et III}

A la surface des cellules du système immunitaire, les RFcyII et III ont une distribution tissulaire différente [10]. Les macrophages, les mastocytes, les neutrophiles et certains 


\section{RÉFÉRENCES}

1. Weinshank RL, Luster AD, Ravetch JV. Function and regulation of a murine macrophage-specific IgG Fc receptor, Fc $\gamma$ R- $\alpha$. J Exp Med 1988; 167 : 1909-21.

2. Ravetch JV, Luster AD, Weinshank R, Pavlovec A, Portnoy DA, Hulmes J, Pan YCE, Unkeless JC. Structural heterogeneity and functional domains of murine immunoglobulin G Fc receptors. Science 1986; 234: 718-23.

3. Brooks DG, Qiu WQ, Luster AD, Ravetch JV. Structure and expression of human IgG FcRII (CD32). Functional heterogeneity is encoded by the alternatively spliced products of multiple genes. $J$ Exp Med 1989 ; 170 : 1369-82.

4. Ra C, Jouvin ME, Kinet J. Complete structure of the mouse mast cell receptor for IgE (FceRI) and surface expression of chimeric receptors (rat-mouse-human) on transfected cells. J Biol Chem 1989; 264 : 15323-30.

5. Koyasu S, D'Adamio L, Arulanandam ARN, Abraham S, Clayton LK, Reinherz EL. T cell receptor complexes containing FcERI $\gamma$. Homodimers in lieu of CD3 $\zeta$ and CD3 $\eta$. Components: a novel isoform expressed on large granular lymphocytes. $J$ Exp Med 1992 ; 175: 203-17.

6. Kurosaki T, Gander I, Wirthmueller U, Ravetch JV. The $\beta$ subunit of the FcERI is associated with the Fc $\gamma$ RIII on mast cells. $J$ Exp Med 1992; 175 : 447-61.

7. Ravetch JV,Perussia B. Alternative membrane forms of FcyRIII (CD16) on human NK cells and neutrophils: cell-type specific expression of two genes which differ in single nucleotide substitutions. $J$ Exp Med 1989 ; 170 : 481-95.

8. Orloff DG, Ra C, Frank SJ, Klausner RD, Kinet JP. Family of disulphide-linked dimers containing the $\zeta$ and $\eta$ chains of the T-cell receptor and the $\gamma$ chain of the Fc receptors. Nature 1990; 347: 189-91.

9. Simmons D, Seed B. The Fc $\gamma$ receptor of natural killer cells is a phospholipidlinked membrane protein. Nature 1988; 333 : $568-70$

10. Daëron $M$, Sautès $C$, Bonnerot $C$, Blank U, Varin N, Even J, Hogarth PM, Fridman WH. Murine type II Fc $\gamma$ receptors and IgG-binding factors. Chem Immunol $1989 ; 47: 21-50$

11. Anderson CL. Structural and functional polymorphism of human FC receptors for IgG. Chem Immunol 1989; 47: 1-27.

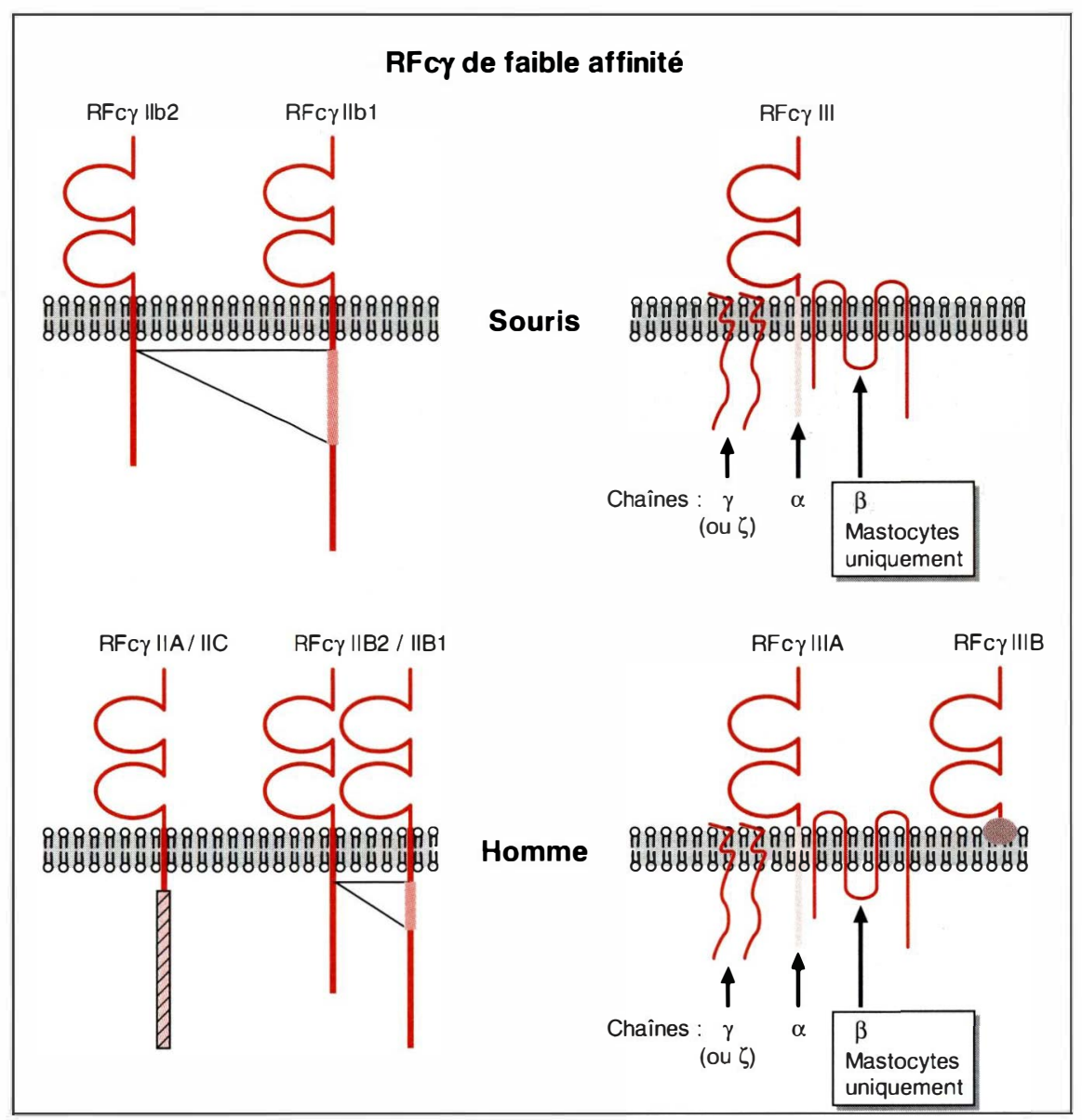

Figure 1. Les récepteurs humains et murins pour la portion Fc des IgG. Les récepteurs sont représentés de manière schématique afin de montrer la forte homologie des régions extracellulaires par opposition à la diversité des régions transmembranaires et intracytoplasmiques. Les récepteurs RFcyll sont monocaténaires et présentent des homologies très importantes; RFcyllb1 et RFcyllb2 ne diffèrent que par une insertion dans le domaine cytoplasmique. Les récepteurs RFcyll sont des complexes multimoléculaires associant 2 ou 3 polypeptides selon les cellules dans lesquelles ils s'expriment, ce qui leur confère une grande diversité fonctionnelle [31].

lymphocytes pré-B coexpriment les RFc $\gamma$ II et III. En revanche, les lymphocytes $\mathrm{T}$ et $\mathrm{B}$ n'expriment que les RFcyII bl, tandis que les cellules NK n'expriment que les RFcyIII. Les complexes immuns ou les immunoglobulines agrégées sur des particules ou des cellules sont les ligands naturels des RFc $\gamma$ II et III. L'interaction des RFc $\gamma$ avec leurs ligands déclenche des phénomènes biologiques de deux ordres. Tout d'abord, la liaison des complexes immuns aux RFc $\gamma$ peut provoquer l'activation de la cellule dont les conséquences biologiques sont varia- bles en fonction du type cellulaire. Ces mêmes complexes immuns peuvent être internalisés via les RFc $\gamma$ puis dégradés.

\section{RFc $\gamma$ et activation cellulaire}

Les conséquences de l'interaction des complexes immuns ou d'autres particules recouvertes d'IgG, via les $\mathrm{RFc} \gamma$, avec les cellules du système immunitaire sont variées et dépendent principalement du type cellulaire. Ainsi, à la surface des macrophages, des neutrophiles et des cel- 


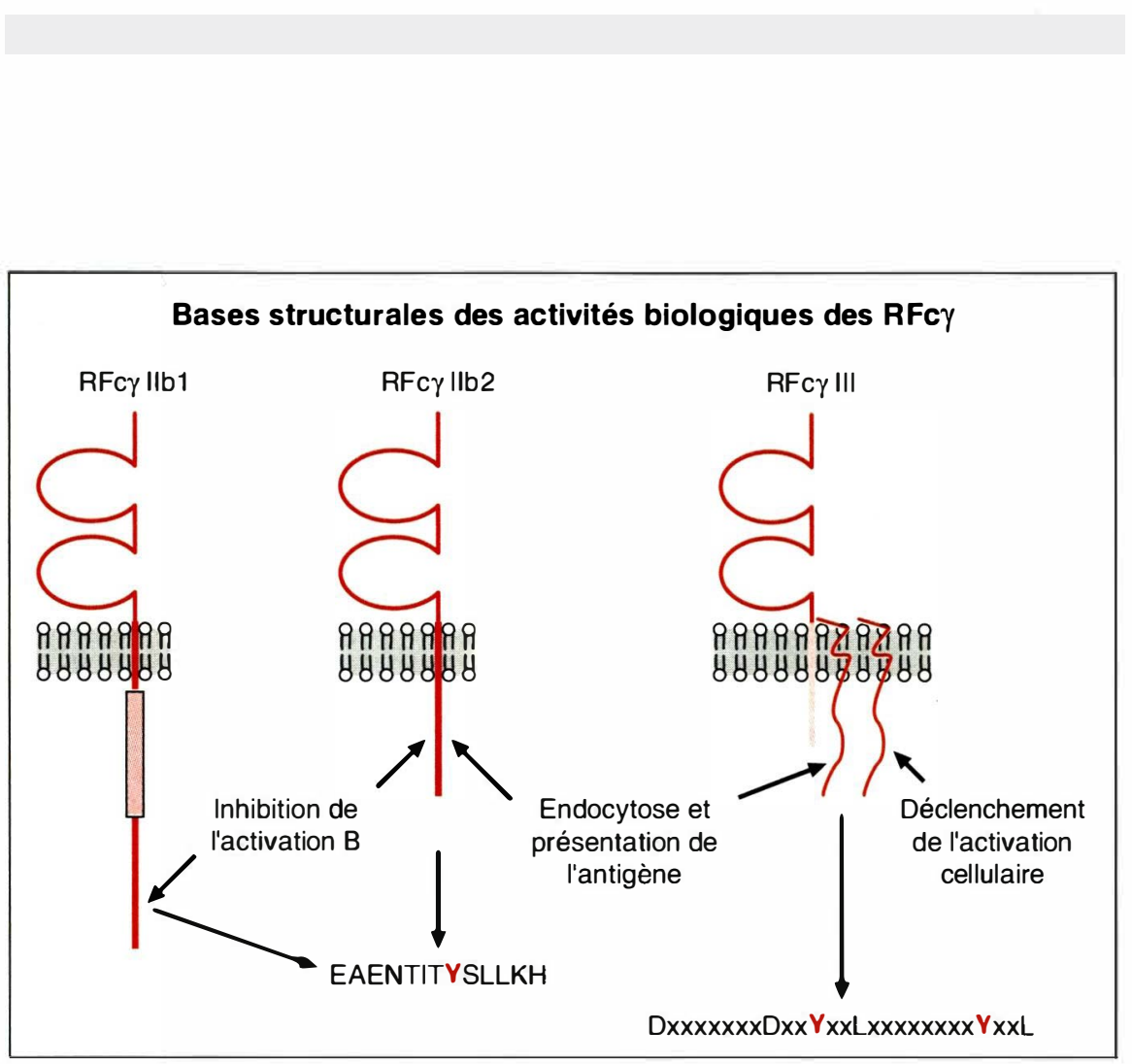

Figure 2. Bases stucturales des activités biologiques des RFc $\gamma$ murins. Pour chacune des trois formes de récepteurs, sont indiquées les régions responsables des différentes activités biologiques. Les RFcyllb1 et les immunoglobulines membranaires sont pontés par les complexes immuns sur les lymphocytes $B$ et bloquent leur activation. Une séquence peptidique du domaine cytoplasmique des RFcyll relaie ce processus. Les RFcyllb2 et RFcyll internalisent des complexes immuns et facilitent la présentation de l'antigène qu'ils contiennent. Ces deux récepteurs sont impliqués aussi dans des phénomènes d'activation cellulaire. Les deux tyrosines présentes dans le domaine cytoplasmique de la chaîne $\gamma$ de RFcyll déterminent à la fois l'endocytose et l'activation cellulaire.Les séquences peptidiques sont représentées dans le code international à une lettre $: A=A l a ; D=A s p ; E=G l u$; $H=$ His $; I=I l e ; K=L y s ; L=L e u ; N=A s n ; S=S e r ; T=T h r ; Y=T y r ;$ $X=n^{\prime i m p o r t e}$ quel acide aminé.

lules NK, les RFc $\gamma$ interviennent dans des phénomènes de cytotoxicité dépendante d'anticorps (antibody dependent cellular cytotoxicity, ADCC) [11]. A la surface des mastocytes, les RFc $\gamma$, comme les RFcEI, sont capables de déclencher la dégranulation de ces cellules et donc aussi la libération de médiateurs de l'inflammation pouvant intervenir dans les phénomènes d'allergie [12]. Enfin, à la surface des lymphocytes $\mathrm{B}$, le pontage des immunoglobulines membranaires avec les RFc $\gamma$ bloque l'activation cellulaire et module ainsi la synthèse $\mathrm{m} / \mathrm{s} n^{\circ} 11$ vol. 9 , novembre 93

\section{Les RFcy III déclenchent I'activation cellulaire}

Les mastocytes et les cellules NK sont deux types cellulaires particulièrement intéressants pour essayer de comprendre lequel des RFc $\gamma$ II et RFcyIII est impliqué dans le déclenchement des phénomènes d'activation cellulaire.

Tout d'abord les cellules NK, chez l'homme comme chez la souris, n'expriment que le RFc $\gamma$ III. Dans les deux espèces, ces récepteurs déclenchent une rapide mobilisation des stocks calciques, la production d'inositol 1,4,5 triphosphate (IP3) et l'activation d'une protéine kinase $\mathrm{C}$ [15], ainsi que l'induction de la transcription des gènes du récepteur de l'interleukine 2 et d'autres lymphokines comme l'interféron $\gamma$ et le TNF $\alpha$ [16]. La libération de médiateurs via le RFcEI et les RFc $\gamma$ des mastocytes fournit des indications sur les bases moléculaires de la signalisation intracellulaire via les RFc $\gamma$. En effet, le RFcEI et le RFc $\gamma$ III ne diffèrent, à la surface des mastocytes, que par la chaîne qui fixe le ligand, la chaîne $\alpha[6,17]$. Ils partagent tous les deux les mêmes chaînes associées: la chaîne $\beta$ et la chaîne $\gamma$, suggérant en conséquence que les voies intracytoplasmiques de transmission d'un signal sont similaires.

Une réponse définitive à la question du rôle respectif des récepteurs de type II et III dans les voies d'activation cellulaire a été apportée grâce à l'utilisation des techniques de biologie moléculaire et cellulaire. Ces différents récepteurs ont été exprimés par transfection à la surface de cellules immunocompétentes comme le lymphome B IIAl.6 [18] et la lignée mastocytaire $\operatorname{RBL}[19,20]$. Dans les deux modèles expérimentaux, les conclusions ont été les mêmes : seuls les RFcyIII sont capables de déclencher des phénomènes d'activation cellulaire dont les conséquences sont variables en fonction du type cellulaire: sécrétion de lymphokines par les lymphocytes [18], libération de sérotonine ou de TNF par les mastocytes [19, 20]. Une analyse moléculaire, utilisant des récepteurs mutants ou chimériques, a établi que la région intracy- 


\section{RÉFÉRENCES}

12. Daëron M, Prouvost-Danon A Voisin $\mathrm{GA}$. Mast cell membrane antigens and $\mathrm{Fc}$ receptors in anaphylaxis. II. Functionally distinct receptors of $\operatorname{IgG}$ and $\operatorname{IgE}$ on mouse mast cells. Cell Immunol 1980 ; 49 : 178-86.

13. Abbas AK, Unanue ER. Interrelationships of surface immunoglobulin and Fc receptors on mouse B lymphocytes. J Immunol 1975 ; 115 : 1665-71

14. Phillips NE, Parker DC. Cross-linking of B lymphocyte Fc $\gamma$ receptors and membrane immunoglobulin inhibits antiimmunoglobulin-induced blastogenesis. $J$ Immunol 1984 ; 132 : 627-35.

15. Carsatella MA, Anegon I, Cuturi M, Griskey P, Trinchieri G, Perussia B. Fc $\gamma$ R (CD16) interaction with ligand induces $\mathrm{Ca}^{2+}$ mobilization and phosphoinositide turnover in human natural killer cells. Role of $\mathrm{Ca}^{2+}$ in $\mathrm{Fc} \gamma \mathrm{R}$ (CD16)-induced transcription and expression of lymphokine genes. J Exp Med 1989; 169: 549-61.

16. Anegon I, Cuturi MC, Trinchieri G, Perussia $\mathrm{B}$. Interaction of $\mathrm{Fc}$ receptor (CD16) ligands induces transcription of interleukin 2 receptor (CD25) and lymphokine genes and expression of their products in human natural killer cells. J Exp Med 1988; 167 : 452-65.

17. Kinet JP. Antibody-cell interactions : Fc receptors. Cell 1989; 57 : 351-4.

18. Bonnerot C, Amigorena S, Choquet D, Choukroun V, Pavlovitch R, Fridman WH. Role of associated $\gamma$-chain in tyrosine kinase activation $\theta 1 \alpha$ murine Fc $\gamma$ RIII. EMBO J 1992 ; 11 : 2747-57

19. Daëron $M$, Bonnerot $C$, Latour $S$, Fridman WH. Murine recombinant Fc $\gamma$ RIII, but not FcyRII, trigger serotonin release in rat basophilic leukemia cells. I Immunol $1992 ; 149$ : 1365-72.

20. Latour S, Bonnerot C, Fridman WH, Daëron $M$. Induction of tumor necrosis factor- $\alpha$ production by mast cells $\theta \imath \alpha$ Fc $\gamma$ R. Role of the Fc $\gamma$ RIII $\gamma$ subunit. J Immunol 1992 ; 149 : 2155-62.

21. Amigorena S, Bonnerot C, Choquet D, Fridman WH, Teillaud JL. FcyRII expression in resting and activated B lymphocy- toplasmique de la chaîne $\gamma$ du RFc $\gamma$ III, qui permet son couplage à des tyrosine kinases, était nécessaire et suffisante à cette activité. Le signal moléculaire de reconnaissance a pu être circonscrit par mutagenèse autour de deux résidus tyrosine (figure 2). Au cours d'autres études, le RFcyIIA humain s'est avéré être également un récepteur capable de déclencher l'activation cellulaire.

\section{Les RFcy II bloquent l'activation des lymphocytes B via les immunoglobulines}

Le cas des lymphocytes B est particulièrement intéressant puisque, d'une part, ces cellules n'expriment que des RFcyIIbl [21] et, d'autre part, leurs fonctions à la surface de ces cellules sont spécifiques de ce type cellulaire. Ainsi, la liaison d'un antigène aux immunoglobulines membranaires d'un lymphocyte B déclenche l'activation puis la différenciation de ces cellules, aboutissant à l'apparition de plasmocytes qui sécrètent des immunoglobulines spécifiques de cet antigène. En revanche, si ce même antigène est complexé avec des anticorps, les immunoglobulines de membrane et le RFcyIIbl sont pontés par les complexes immuns, bloquant du même coup l'activation du lymphocyte B [13]. Cette fonction du RFcyllbl est très importante puisqu'elle permet de contrôler la réponse du système immunitaire contre un antigène donné. Quand les complexes immuns sont en excès par rapport aux antigènes libres, ils peuvent ainsi exercer une régulation négative sur la synthèse des anticorps. In vitro, cette interaction bloque certains phénomènes biologiques déclenchés par les immunoglobulines membranaires comme la mobilisation des stocks calciques, la production d'IP3 [22], l'activation de protéine kinases ou encore la sécrétion de lymphokines [23].

L'implication des seuls RFc $\gamma$ II dans l'inhibition de l'activation des lymphocytes B a été, là encore, clairement démontrée en utilisant la même approche associant les techniques de biologie moléculaire et cellulaire. Une séquence peptidique de quelques acides aminés, localisée dans la région intracytoplasmique des RFcyII, s'est avérée déterminante dans ces processus d'inhibition [24] (figure 2). Ces résultats posent d'une manière générale la question du rôle des RFcyII dans les mécanismes de contrôle des voies d'activation cellulaire. Existe-t-il d'autres molécules membranaires dont les mécanismes de signalisation peuvent être bloqués par le pontage aux RFc $\gamma \mathrm{II}$ ? Et quel est le rôle du RFc $\gamma$ II dans d'autres types de réponses immunitaires, passant par exemple par le récepteur de l'antigène des lymphocytes $\mathrm{T}$ ou encore par le récepteur de forte affinité pour les $\operatorname{IgE}$ des mastocytes?

\section{RFcy et endocytose}

L'interaction des RFc $\gamma$ avec leurs ligands provoque l'internalisation du complexe puis sa dégradation. Les cellules impliquées dans ces phénomènes d'internalisation via les $\mathrm{RFc} \gamma$ sont principalement les macrophages qui coexpriment les RFc $\gamma$ II et RFc $\gamma$ III. Ils déterminent, à la surface de ces cellules, la phagocytose de particules recouvertes d'immunoglobulines ou l'endocytose de particules plus petites, comme des complexes immuns. Ces récepteurs participent donc à la clairance des complexes immuns et à l'internalisation d'un antigène présent dans ces complexes.

La clairance des complexes immuns a surtout été étudiée chez l'homme puisque son dysfonctionnement est associé à certaines maladies autoimmunes comme le lupus érythémateux disséminé, le syndrome de Gougerot-Sjögren et la dermatite herpétiforme [25]. Dans le cas du lupus érythémateux disséminé, les taux élevés de complexes immuns circulants ont pu être rattachés à une diminution de la phagocytose de ces complexes via les RFc $\gamma$ des macrophages [26]. Dans l'hémoglobinurie paroxystique nocturne, un déficit en RFc $\gamma$ à la surface des neutrophiles est corrélé à la sévérité de la maladie et à une susceptibilité accrue à certaines infections bactériennes [27].

La présentation de l'antigène est une autre conséquence biologique 
de l'internalisation de complexes immuns. En effet, quand un antigène est internalisé, celui-ci est rapidement dégradé puis les peptides dérivés s'associent aux molécules de classe II du CMH. Les complexes classe II-peptide sont acheminés à la surface de la cellule où ils sont présentés aux cellules $\mathrm{T}$ auxiliaires qui les reconnaissent par leur récepteur de l'antigène, le TcR. Les RFc $\gamma$ des macrophages, en facilitant l'internalisation d'un antigène quand il est complexé aux IgG, augmentent l'efficacité de ce phénomène de présentation antigénique aux lymphocytes $T$ [28]. Les conséquences biologiques ou immunologiques de l'internalisation des ligands des RFc $\gamma$ sont donc multiples, mais les mécanismes déterminant l'endocytose de ces récepteurs restent à élucider.

\section{Endocytose \\ des complexes immuns via les RFcy}

Les cellules ayant une forte capacité phagocytaire comme les macrophages coexpriment les RFc $\gamma$ II et RFcyIII [2], ce qui rend impossible leur utilisation pour une étude structurale des différents RFc $\gamma$ impliqués dans l'endocytose de complexes immuns. Il y a quelques années, les seules lignées $\operatorname{RFc} \gamma \mathrm{II}(-)$ facilement transfectables étaient d'origine fibroblastique. En exprimant les deux isoformes de RFc $\gamma$ II à la surface des cellules $\mathrm{L}$ ou des cellules $\mathrm{CHO}$, il a été montré que seul le RFcyllb2 entraîne l'endocytose des complexes immuns par l'intermédiaire des vésicules recouvertes de clathrine. Au contraire, l'agrégation des RFc $\gamma I I b 1$ par les complexes immuns déclenche la liaison du récepteur au cytosquelette de la cellule, empêchant ainsi son internalisation.

Une analyse systématique du rôle des différentes formes de RFc $\gamma$, dans les phénomènes d'internalisation et dans la présentation d'un antigène complexé à des anticorps, a pu être réalisée grâce à l'amélioration des techniques de transfection qui a permis d'exprimer ces récepteurs à la surface de cellules immunocompétentes comme les cellules de lymphome B IIAl.6. Nous avons ainsi montré que le RFcyllb2 et le RFcyIII internalisent des complexes immuns et facilitent la présentation de l'antigène qu'ils contiennent [24, 29]. Si cette propriété commune les rapproche, certaines différences suggèrent que les mécanismes permettant l'endocytose des deux récepteurs sont différents. L'internalisation du RFcyIIb2 requiert un signal localisé dans sa région intracytoplasmique [24] tandis que l'internalisation du RFc $\gamma I I I$ est indépendante de la région intracytoplasmique de la chaîne qui fixe le ligand [29]. En revanche, l'endocytose du RFcyIII requiert la coexpression de la chaîne $\gamma$ dont le signal d'internalisation est localisé dans sa région intracytoplasmique (figure 2). Les signaux d'internalisation des deux récepteurs sont différents. Le signal présent dans la région intracytoplas-

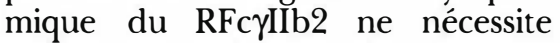
qu'une seule tyrosine, alors que celui présent dans la chaîne $\gamma$ requiert, pour être actif, l'intégrité de deux tyrosines distantes de dix acides aminés.

Globalement, ces deux récepteurs sont impliqués non seulement dans l'endocytose de complexes immuns mais aussi dans des phénomènes d'activation cellulaire. Le RFc $\gamma$ IIb2, ponté aux IgG de membrane, bloque l'activation des cellules via ces molécules. A l'opposé, l'agrégation du RFc $\gamma I I I$, mais non celle du RFc $\gamma I I b 2$, peut déclencher l'activation de tyrosine kinases. La même séquence peptidique du RFc $\gamma$ IIb2 comporte le signal qui détermine l'internalisation et l'inhibition de l'activation via ce récepteur, mais le résidu tyrosine est impliqué uniquement dans les phénomènes d'endocytose. En revanche, les deux tyrosines présentes dans le domaine intracytoplasmique de la chaîne $\gamma$ déterminent non seulement l'endocytose [29] mais aussi le déclenchement de l'activation cellulaire via le RFc $\gamma I I I$ [18]. Ainsi, un même signal semble responsable de deux activités distinctes du RFc $\gamma$ III dont l'une, au moins, implique l'activation d'une tyrosine kinase. Un signal similaire a été décrit dans la région intracytoplasmique des différentes chaînes du complexe CD3 duTcR [30] mais on ne sait pas encore s'il est responsable de l'internalisation de ce récepteur.
Les deux activités du RFc $\gamma$ III sontelles reliées ou, au contraire, indépendantes mais déterminées par la même région du domaine intracytoplasmique de la chaîne $\gamma$ ? Quelle est la participation de l'activation de tyrosine kinases dans le déclenchement de l'endocytose du RFcyIII ou le routage du récepteur à l'intérieur de la cellule? Toutes ces questions sont encore sans réponse et mettent en évidence la nécessité de caractériser les différentes étapes de l'internalisation d'un antigène via les RFc $\gamma$.

\section{Rôle de la présentation de l'antigène via les RFcy}

Quels que soient les mécanismes d'internalisation des RFc $\gamma$ IIb2 et RFcyllI, nous avons montré que, globalement, les conséquences de l'endocytose de complexes immuns, via ces deux récepteurs, sont les mêmes et permettent une facilitation de la présentation de l'antigène $[24,29]$. Les RFc $\gamma$ sont exprimés par deux types cellulaires qui présentent un antigène associé aux molécules de classe II du CMH, les lymphocytes B et les macrophages.

Dans les lymphocytes B, la facilitation de la présentation de l'antigène via l'internalisation de complexes immuns par des RFc $\gamma$ pourrait être à l'origine de certaines maladies. Grâce à des complexes immuns, n'importe quel antigène peut être internalisé efficacement puis présenté à la surface d'un lymphocyte B d'une autre spécificité antigénique, dans la mesure où il exprime le RFc $\gamma$ IIb2 ou le RFc $\gamma$ III. Ainsi, l'endocytose d'un complexe immun aboutit à l'activation puis à la différenciation de ce lymphocyte $B$ qui va produire des anticorps contre un autre antigène que celui contenu dans le complexe immun. Si celuici est un antigène du soi, les anticorps produits seront des autoanticorps pouvant être à l'origine de maladies auto-immunes. Heureusement, la plupart des lymphocytes B mûrs expriment uniquement le RFcyllbl qui est incapable d'endocytose avec son ligand, et ne permet donc pas ce type de phénomène. Mais nous avons observé que, dans certaines conditions, le RFc $\gamma$ III ou le 


\section{RÉFÉRENCES}

22. Cambier JC, Ransom JT. Molecular mechanisms of transmembrane signaling in B lymphocytes. Ann Rev Immunol 1987; 5 : 175-98.

23. Justement LB, Kreiger J, Cambier JC. Production of multiple lymphokines by the A20.1 B cell lymphoma af ter cross-linking of membrane Ig by immunobilized anti-Ig. J Immunol 1989 ; 43 : 881-8.

24. Amigorena S, Bonnerot C, Drake JR, Choquet D, Hunzinker W, Guillet JG, Webster P, Sautes C, Mellman I, Fridman WH. Cytoplasmic domain heterogeneity and functions of IgG Fc receptors in Blymphocytes. Science 1992 ; 256: 1808-12.

25. Lawley TJ, Hall RP, Fauci AS, Katz SI, Hamburger MI, Frank MM. Def ective Fcreceptor functions associated with the HLA-B8/DRw3 haplotype: studies in patients with dermatitis herpetiformis and normal subjects. N Engl J Med 1981; 300 : 185-200.

26. Salmon JE, Kimberley RP, Gibofsky A, Fotino M. Defective mononuclear phagocyte function in systemic lupus erythematosus : dissociation of $F c$ receptor-ligand binding and internalization. I Immunol 1984 ; 133 : 2525-32.

27. Selvaraj P, Rosse F, Silber R, Springer TA. The major FC receptor in blood has a phosphatidylinositol anchor and is deficient in paroxysmal nocturnal haemoglobinuria. Nature 1988; 333: 565-7.

28. Manca F, Fenoglio D, Li Pira G, Kunkl A, Celada F. Effect of antigen/antibody ratio on macrophage uptake, processing, and presentation to T cells of antigen complexed with polyclonal antibodies. J Exp Med 1991; 173: 37-51.

29. Amigorena S, Salamero J, Davoust J, Fridman, WH, Bonnerot C.Tyrosinecontaining motif that transduces cell activation signals also determines internalisation and antigen presentation via type III receptors for IgG. Nature, 1992; 358 : $337-41$.

30. Letourneur F, Klausner, RD. Activation of $\mathrm{T}$ cells by a tyrosine kinase activation Domain in the cytoplasmic tail of CD3E. Science 1992; 255 : 79-81.

31. Rochet N, Anderson P, Vivier E. Structure et fonction du complexe CD16: $\zeta: \gamma$ des cellules NK médecine/sciences $1992 ; 8$ : 359-65.
RFcyIIb2 peuvent s'exprimer à la surface de cellules de la lignée $B$. Le RFcyIII est présent sur certains lymphocytes pré-B [4], et l'interleukine 4 induit l'expression du RFcyIIb2 sur une lignée B. Cela suggère qu'une modification des conditions d'activation de certains lymphocytes B pourrait induire l'expression de l'un ou l'autre de ces récepteurs et donner lieu ainsi à des maladies auto-immunes.

Les macrophages coexpriment le RFc $\gamma I I b 2$ et RFcyIII mais dans une proportion relative qui peut dépendre de l'état d'activation des cellules. Des macrophages péritonéaux expriment majoritairement le RFc $\gamma$ IIb2 mais un traitement par l'interféron $\gamma$ augmente l'expression du RFc $\gamma$ III et diminue celle du RFcyIIb2. En fonction de l'état d'activation de la cellule, l'internalisation des complexes immuns va donc passer préférentiellement par un récepteur ou par l'autre. Si, globalement, les deux phénomènes aboutissent à la présentation de l'antigène, il est probable qu'au moment de l'agrégation des récepteurs par la fixation des complexes immuns les phénomènes intracellulaires induits sont différents. En effet, quand le RFcylII est présent, la phase d'activation, secondaire à la présentation de l'antigène, est précédée d'une étape directe d'activation cellulaire liée à l'agrégation des RFc $\gamma$ III. La sécrétion de lymphokines induite dans ce cas pourrait intervenir dans les interactions entre le lymphocyte $\mathrm{T}$ et le macrophage, ou modifier le devenir de la particule ingérée dans la cellule.

En conclusion, les RFc $\gamma$ II et RFc $\gamma$ III ont une structure différente et s'expriment à la surface de cellules du système immunitaire où des facteurs variés peuvent avoir des effets opposés sur leur expression. Ainsi, ces résultats semblent indiquer que la fixation d'un même ligand sur chacun des trois $\mathrm{RF} c \gamma$ pourrait avoir des conséquences biologiques différentes, dépendant non seulement du récepteur mais aussi du type cellulaire dans lequel il s'exprime. L'étude de la spécificité fonctionnelle des trois formes de RFc $\gamma$, ainsi que l'analyse des bases structurales qui déterminent ces différentes acti- vités, ont permis de mieux préciser leurs fonctions à la surface de cellules du système immunitaire

\section{Summary}

Structural basis of functional heterogeneity of the Fc receptors

The low-affinity receptors for the $\mathrm{Fc}$ portion of $\operatorname{IgG}(\mathrm{Fc} \gamma \mathrm{R})$ are expressed on most of the cells of the immune system. They bind immune complexes but not monomeric IgG. These receptors are a family of surface glycoproteins with homologous extracellular domains and different transmembrane and cytoplasmic portion. They mediate the biological activities of antibodies bound at the surface of all the immune cells : Fc $\gamma$ Rs activate macrophages, neutrophils and NK cells, participating in antibody-dependent cellular cytotoxicity; Fc $\gamma$ Rs activate mast cells, resulting in the release of inflammation mediators and the induction of cytokines; in B cells, on the contrary, cross-linking of Fc $\gamma \mathrm{R}$ and IgM blocks cellular activation, inhibiting IgG production. $\mathrm{Fc} \gamma \mathrm{Rs}$ are also involved in ligand internalization and the clearing of immune complexes.

\section{SOCIÉTÉ DE BIOLOGIE}

Séance du 15 décembre 1993 Amélioration des plantes Yves Demarly

L'amélioration des plantes, stratégie, problèmes actuels, espoirs et réalités

Yvonne Cauderon (Membre de l'Académie d'Agriculture) Cytogénétique et amélioration des pantes: quelques exemples Emmanuel Picard

Haploïdisation et amélioration des plantes

Michel Caboche (INRA, Versailles) Le transfert de gènes: utilisation de la transgénèse pour l'analyse du métabolisme du nitrate chez les plantes supérieures Vincent Petiard (Francereco-Nestlé) Biotechnologies végétales, exemples d'application.

La séance aura lieu à 16 h 30 à l'Hôpital

Sainte-Anne, Amphithéâtre du Service du Professeur Loo, 7, rue Cabanis, 75014 Paris 